Proceedings of the Edinburgh Mathematical Society (2003) 46, 649-668 (C)

DOI:10.1017/S0013091502000834 Printed in the United Kingdom

\title{
$B$-CONVEX OPERATOR SPACES
}

\author{
JAVIER PARCET \\ Department of Mathematics, Universidad Autónoma de Madrid, \\ Madrid 28049, Spain (javier.parcet@uam.es)
}

(Received 3 September 2002)

\begin{abstract}
The notion of $B$-convexity for operator spaces, which a priori depends on a set of parameters indexed by $\Sigma$, is defined. Some of the classical characterizations of this geometric notion for Banach spaces are studied in this new context. For instance, an operator space is $B_{\Sigma}$-convex if and only if it has $\Sigma$-subtype. The class of uniformly non- $\mathcal{L}^{1}(\Sigma)$ operator spaces, which is also the class of $B_{\Sigma}$-convex operator spaces, is introduced. Moreover, an operator space having non-trivial $\Sigma$-type is $B_{\Sigma}$-convex. However, the converse is false. The row and column operator spaces are nice counterexamples of this fact, since both are Hilbertian. In particular, this result shows that a version of the Maurey-Pisier Theorem does not hold in our context. Some other examples of Hilbertian operator spaces will be considered. In the last part of this paper, the independence of $B_{\Sigma}$-convexity with respect to $\Sigma$ is studied. This provides some interesting problems, which will be posed.
\end{abstract}

Keywords: $B_{\Sigma}$-convex operator space; uniformly non- $\mathcal{L}^{1}(\Sigma)$ operator space; non-trivial $\Sigma$-type; $K_{\Sigma}$-convex operator space; row and column operator spaces

2000 Mathematics subject classification: Primary 46L07

Secondary $42 \mathrm{C} 15$

\section{Introduction}

In the last 30 years, the notions of type and cotype of a Banach space with respect to several orthonormal systems have been deeply investigated. It turns out that, in order to study some geometric properties of the Banach space considered, these notions are very useful. In a series of papers with José García-Cuerva and José Manuel Marco, we have initiated a theory of type and cotype for operator spaces. As might be expected, the question is to what extent these notions are related to the geometry of operator spaces.

Our previous results can be summarized as follows. In [3], we study the validity of the Hausdorff-Young inequality for vector-valued functions defined on a non-commutative compact group. This inequality does not make sense when our functions take values in a Banach space, we need an operator space structure on it. This requirement goes back to Pisier's work [14], where non-commutative vector-valued Lebesgue spaces are studied. In particular, this gives rise to the notions of Fourier type and cotype of an operator space with respect to a non-commutative compact group. We investigate in [3] the basic properties of this notion. The paper [4] is devoted to the study of the sharp Fourier type and cotype exponents of Lebesgue spaces and Schatten classes. This is basic 
in the commutative theory. However, the problem of finding the sharp exponents of a given operator space is highly non-trivial, even for the simplest case of Lebesgue spaces. We have solved part of this problem when dealing with compact semisimple Lie groups in [4] using the well-developed representation theory on this kind of group. Finally, [2] deals with the general theory of type and cotype for operator spaces. First, we define the notion of quantized orthonormal system, which plays the role of the orthonormal system in the classical setting. Then we introduce the notions of type and cotype with respect to such a system. In particular, this provides non-commutative extensions of the notions of Rademacher, Steinhaus and Gaussian type and cotype. The main result in [2] is an operator space version of the classical result of Kwapien [7], which characterizes Hilbert spaces up to isomorphism by means of vector-valued orthogonal series. We give several approaches to this result in the operator space setting, characterizing in such a way $\mathrm{OH}$ operator spaces up to complete isomorphism by a type $2 /$ cotype 2 condition.

These previous results show that, also in the non-commutative setting, there exists some interaction between the theory of type and cotype and the geometry of operator spaces. Hence, it seems that the next step in the process should be to study the notion of $B$-convex operator space. Interest in $B$-convexity for Banach spaces was generated by $[\mathbf{1}]$, where Beck studied certain strong laws of large numbers for Banach space valued random variables. However, our motivation to study this notion for operator spaces lies in some other characterizations of this geometric condition. For instance, the following are equivalent for any Banach space $B$ :

(a) $B$ is $B$-convex;

(b) $B$ does not contain $l_{n}^{1}$ uniformly;

(c) $B$ has Rademacher subtype;

(d) $B$ has non-trivial Rademacher type;

(e) $B$ is $K$-convex.

Giesy proved in [5] the equivalence between (a) and (b), while Pisier proved the others. The proof of the equivalence between (a), (c) and (d) can be found in [11]. Finally, the equivalence between $B$-convexity and $K$-convexity was given in [12] and is much more complicated. For some other characterizations of $B$-convexity, the reader is referred to [10]. Our aim is to study the validity of these classical characterizations for operator spaces.

We shall follow a notation similar to that employed in [2]. Namely, let $(\Omega, \mathcal{M}, \mu)$ be a probability measure space with no atoms and let $\boldsymbol{d}_{\Sigma}=\left\{d_{\sigma}: \sigma \in \Sigma\right\}$ be a family of positive integers indexed by $\Sigma$. The quantized Steinhaus system of parameters $\left(\Sigma, \boldsymbol{d}_{\Sigma}\right)$ is a collection $\mathcal{S}_{\Sigma}=\left\{\zeta^{\sigma}: \Omega \rightarrow U\left(d_{\sigma}\right)\right\}_{\sigma \in \Sigma}$ of independent random unitary matrices, uniformly distributed on the unitary group $U\left(d_{\sigma}\right)$ equipped with its normalized Haar measure $\lambda_{\sigma}$. Such a system led us to the notion of $\Sigma$-type. There is a similar definition for the quantized Rademacher system of parameters $\left(\Sigma, \boldsymbol{d}_{\Sigma}\right)$. However, since we work with operator spaces (which are defined over the complex field), it will be more convenient to deal 
with the Steinhaus system. This does not make any difference since, as was mentioned in $[\mathbf{2}]$, Rademacher and Steinhaus $\Sigma$-type are equivalent notions.

The organization of the paper is as follows. In $\S 2$ we define the main notions we shall work with, such as $B_{\Sigma}$-convexity, $\Sigma$-type, $\Sigma$-subtype and uniformly non- $\mathcal{L}^{1}(\Sigma)$ operator spaces. We also prove some basic results which will be applied throughout the paper. Some of these notions are equivalent. Namely, an operator space has $\Sigma$-subtype if and only if it is uniformly non- $\mathcal{L}^{1}(\Sigma)$ and each of these properties are equivalent to the

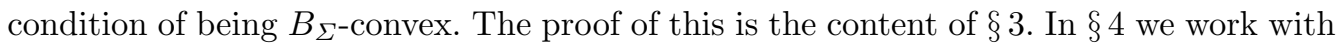
certain tensor submultiplicativity which generalizes the classical submultiplicativity of the constants involved in the theory (see $[\mathbf{1 1}]$ for the details). This will be useful in order to see the validity in our context, for the main sets of parameters $\left(\Sigma, \boldsymbol{d}_{\Sigma}\right)$, of some wellknown classical results. In $\S 5$ we show that the property of having non-trivial $\Sigma$-type is stronger than $B_{\Sigma}$-convexity. Moreover, we provide examples of $B_{\Sigma}$-convex Hilbertian operator spaces failing the non-trivial $\Sigma$-type condition. Some other interesting examples will be treated. Finally, $\S 6$ is devoted to the study of the dependence of $B_{\Sigma}$-convexity with respect to $\left(\Sigma, \boldsymbol{d}_{\Sigma}\right)$. First, we prove that there is no dependence when we work only with those sets of parameters having $\boldsymbol{d}_{\Sigma}$ unbounded. Then we introduce the notion of $K_{\Sigma}$-convexity, and we show that the independence with respect to $\left(\Sigma, \boldsymbol{d}_{\Sigma}\right)$ is equivalent to seeing that an operator space is $B_{\Sigma}$-convex if and only if it is $K_{\Sigma^{-}}$-convex. Also a sufficient condition for the independence, in terms of the way in which $S_{n}^{1}$ embeds in $S^{2}\left(l^{1}\right)$, is given.

Throughout this paper, some basic notions of operator spaces and vector-valued Schatten classes will be assumed. The main results that we will be using can be found in [14]. Also, the main results in the theory of type for operator spaces will be assumed. The reader is referred to [2] for a brief summary of them. Finally, throughout this work we make a slight abuse of notation since sometimes, when there is no risk of confusion, we shall write $\Sigma$ to denote the set of parameters $\left(\Sigma, \boldsymbol{d}_{\Sigma}\right)$.

\section{The main definitions}

Given an operator space $E$, a set of parameters $\left(\Sigma, \boldsymbol{d}_{\Sigma}\right)$ and $1 \leqslant p<\infty$, we define the spaces $\mathcal{L}_{E}^{p}(\Sigma)$ as follows:

$$
\begin{aligned}
& \mathcal{L}_{E}^{p}(\Sigma)=\left\{A \in \prod_{\sigma \in \Sigma} M_{d_{\sigma}} \otimes E:\|A\|_{\mathcal{L}_{E}^{p}(\Sigma)}=\left(\sum_{\sigma \in \Sigma} d_{\sigma}\left\|A^{\sigma}\right\|_{S_{d_{\sigma}}^{p}(E)}^{p}\right)^{1 / p}<\infty\right\}, \\
& \mathcal{L}_{E}^{\infty}(\Sigma)=\left\{A \in \prod_{\sigma \in \Sigma} M_{d_{\sigma}} \otimes E:\|A\|_{\mathcal{L}_{E}^{\infty}(\Sigma)}=\sup _{\sigma \in \Sigma}\left\|A^{\sigma}\right\|_{S_{d_{\sigma}}^{\infty}(E)}<\infty\right\}
\end{aligned}
$$

where we write $M_{n}$ for the vector space of $n \times n$ complex matrices and $S_{n}^{p}(E)$ stands for the Schatten $p$-class over $M_{n}$ with values in $E$. We shall also use the infinite-dimensional Schatten classes $S^{p}(E)$ with values in $E$, the reader is referred to [14] for a precise definition of these spaces. The case in which $E=\mathbb{C}$ will be denoted by $\mathcal{L}^{p}(\Sigma)$. We impose on $\mathcal{L}_{E}^{p}(\Sigma)$ its natural operator space structure (see [3] or Chapter 2 of $[\mathbf{1 4}]$ for 
the details). Now let $1 \leqslant p \leqslant \infty$ and let $\Gamma$ be any subset of $\Sigma$, if $\zeta^{\sigma}$ are the elements of the quantized Steinhaus system (as defined in $\S 1$ ), then we define the mapping $\mathrm{T}_{p}(\Gamma, E)$ by the relation

$$
A \in \mathcal{L}_{E}^{p}(\Gamma) \mapsto \sum_{\sigma \in \Gamma} d_{\sigma} \operatorname{tr}\left(A^{\sigma} \zeta^{\sigma}\right) \in L_{E}^{2}(\Omega) .
$$

We shall denote $\mathrm{T}_{p}(\Sigma, E)$ by $\mathrm{T}_{p}(E)$. For $\Gamma$ finite, we also define the number $\Delta_{\Gamma}=$ $\sum_{\sigma \in \Gamma} d_{\sigma}^{2}$.

Definition 2.1. Let $1 \leqslant p \leqslant 2$. We say that an operator space $E$ has $\Sigma$-type $p$ if the mapping $\mathrm{T}_{p}(E)$ is completely bounded.

Remark 2.2. This notion of type depends on the choice of the quantized Steinhaus system $\mathcal{S}_{\Sigma}$ we are working with. If we take $\Sigma_{0}=\mathbb{N}$ and $d_{\sigma}=1$ for all $\sigma \in \Sigma_{0}$, then we work with the classical Steinhaus system and Definition 2.1 provides a completely bounded version of the classical definition of type. We shall refer to these parameters as the classical set of parameters $\Sigma_{0}$. One could think that this is the right definition of type in the operator space setting and that there is no reason to introduce all the quantized Steinhaus systems in the theory. But in fact, those quantized Steinhaus systems $\mathcal{S}_{\Sigma}$ with $\boldsymbol{d}_{\Sigma}$ unbounded are sometimes the right systems to work with. One example of this assertion is given in [2], where we show that for those systems we can obtain an operator space version of Kwapień's Theorem with weaker hypotheses than for the classical Steinhaus system. Another example will be given in $\S 6$, where we shall prove that the notion of $B_{\Sigma}$-convexity is independent of $\Sigma$ whenever $\boldsymbol{d}_{\Sigma}$ is unbounded.

Remark 2.3. Let $\mathcal{S}_{\Sigma}^{q}(E)$ be the closure in $L_{E}^{q}(\Omega)$ of the subspace spanned by the entries $\left\{\zeta_{i j}^{\sigma}: \sigma \in \Sigma, 1 \leqslant i, j \leqslant d_{\sigma}\right\}$ of the functions of $\mathcal{S}_{\Sigma}$ with $E$-valued coefficients. A version of the Khintchine-Kahane inequalities for random matrices given in [8] implies that the norm of $\mathcal{S}_{\Sigma}^{q_{1}}(E)$, regarded as a Banach space, is equivalent to that of $\mathcal{S}_{\Sigma}^{q_{2}}(E)$ whenever $1 \leqslant q_{1}, q_{2}<\infty$. In particular, given $1 \leqslant p \leqslant 2$ and $1 \leqslant q<\infty$, the validity of the inequality

$$
\left\|\sum_{\sigma \in \Sigma} d_{\sigma} \operatorname{tr}\left(A^{\sigma} \zeta^{\sigma}\right)\right\|_{L_{E}^{q}(\Omega)} \leqslant c\left(\sum_{\sigma \in \Sigma} d_{\sigma}\left\|A^{\sigma}\right\|_{S_{d_{\sigma}}^{p}(E)}^{p}\right)^{1 / p}
$$

does not depend on the value of $q$. However, for $1 \leqslant q_{1} \neq q_{2}<\infty$, Pisier showed in [14] that in general $\mathcal{S}_{\Sigma}^{q_{1}}(E)$ and $\mathcal{S}_{\Sigma}^{q_{2}}(E)$ are not completely isomorphic as operator spaces with their natural operator space structure. Therefore, in contrast with (2.1), each choice of the exponent $1 \leqslant q<\infty$ in Definition 2.1 gives different notions of $\Sigma$-type.

Remark 2.4. The absence of Khintchine inequalities for operator spaces forces us to choose an exponent $q$ in the definition of $\Sigma$-type. Our choice differs from that of [2]. The reason is that in [2] (where we took $q=p^{\prime}$ in the definition of $\Sigma$-type $p$ ) the aim was to have a unified theory of type and cotype for uniformly bounded quantized orthonormal systems, while here the choice $q=2$ facilitates our work. However, although the notion of $\Sigma$-type given here is not the same as in [2], we shall mainly be concerned 
with the notion of non-trivial $\Sigma$-type for which the choice of $q$ does not matter! Namely, let $1 \leqslant q_{1}<q_{2}<\infty$ and $1<p \leqslant 2$. Let us consider the mapping $\mathrm{T}_{p}^{q}(E)$ given by

$$
A \in \mathcal{L}_{E}^{p}(\Sigma) \mapsto \sum_{\sigma \in \Sigma} d_{\sigma} \operatorname{tr}\left(A^{\sigma} \zeta^{\sigma}\right) \in L_{E}^{q}(\Omega)
$$

The complete boundedness of $\mathrm{T}_{p}^{q_{2}}(E)$ obviously implies the complete boundedness of $\mathrm{T}_{p}^{q_{1}}(E)$. Conversely, let us assume that $\mathrm{T}_{p}^{q_{1}}(E)$ is completely bounded. Then, since the cb-norm of $\mathrm{T}_{1}^{\infty}(E)$ is 1 for any operator space $E$, by complex interpolation there exists some $1<r<p$ such that $\mathrm{T}_{r}^{q_{2}}(E)$ is also completely bounded. That is, the notion of non-trivial $\Sigma$-type does not depend on the choice of $q$ in the definition of $\Sigma$-type.

We also generalize to our context some other notions of the commutative theory. For that we shall need the numbers $\Delta_{\Gamma}$ defined above for each finite $\Gamma \subset \Sigma$. Let $E$ be an operator space and let $\left(\Sigma, \boldsymbol{d}_{\Sigma}\right)$ be any set of parameters. We say that $E$ has $\Sigma$-subtype if there exists a finite subset $\Gamma$ of $\Sigma$ such that

$$
\left\|\mathrm{T}_{2}(\Gamma, E)\right\|_{\mathrm{cb}}<\Delta_{\Gamma}^{1 / 2}
$$

Now we define $B_{\Sigma}$-convex and uniformly non- $\mathcal{L}^{1}(\Sigma)$ operator spaces. As in the previous definitions, when dealing with the classical set of parameters $\Sigma_{0}$, we obtain a completely bounded version of the classical notion.

Definition 2.5. Let $E$ be an operator space and let us fix a set of parameters $\left(\Sigma, \boldsymbol{d}_{\Sigma}\right)$.

(i) $E$ is said to be $B_{\Sigma}$-convex if there exists a finite subset $\Gamma$ of $\Sigma$ and $0<\delta \leqslant 1$ such that, for any family $\left\{A^{\sigma} \in M_{d_{\sigma}} \otimes S^{2}(E)\right\}_{\sigma \in \Gamma}$, we have

$$
\frac{1}{\Delta_{\Gamma}} \inf _{B^{\sigma} \text { unitary }}\left\|\sum_{\sigma \in \Gamma} d_{\sigma} \operatorname{tr}\left(A^{\sigma} B^{\sigma}\right)\right\|_{S^{2}(E)} \leqslant(1-\delta) \max _{\sigma \in \Gamma}\left\|A^{\sigma}\right\|_{S_{d_{\sigma}}^{\infty}\left(S^{2}(E)\right)} .
$$

(ii) $E$ is said to contain $\mathcal{L}^{1}(\Gamma) \lambda$-uniformly if, for each finite subset $\Gamma$ of $\Sigma$, there exists a subspace $F_{\Gamma}$ of $S^{2}(E)$ and a linear isomorphism $\Lambda_{\Gamma}: \mathcal{L}^{1}(\Gamma) \rightarrow F_{\Gamma}$ such that

$$
\left\|\Lambda_{\Gamma}\right\|_{\mathrm{cb}}\left\|\Lambda_{\Gamma}^{-1}\right\| \leqslant \lambda
$$

$E$ is uniformly non- $\mathcal{L}^{1}(\Sigma)$ if it does not contain $\mathcal{L}^{1}(\Gamma) \lambda$-uniformly for some $\lambda>1$.

Remark 2.6. The reader could expect that, in the given definition of containing $\mathcal{L}^{1}(\Gamma) \lambda$-uniformly, we should require that $\left\|\Lambda_{\Gamma}\right\|_{\mathrm{cb}}\left\|\Lambda_{\Gamma}^{-1}\right\|_{\mathrm{cb}} \leqslant \lambda$. However, we have given an intermediate notion between that condition and the classical notion, which uses the Banach-Mazur distance. The reason for that will become clear in Theorem 3.2.

Remark 2.7. For the classical set of parameters $\Sigma_{0}$, the given definition of containing $\mathcal{L}^{1}(\Gamma) \lambda$-uniformly can be rephrased by saying that $S^{2}(E)$ contains $l_{n}^{1} \lambda$-uniformly in the Banach space sense. Namely, the cb-norm of $\Lambda_{\Gamma}$ coincides in this case with its operator norm since it is defined on a max operator space. 
Remark 2.8. We shall see in $\S 3$ that Definition 2.5 does not change if we use $S^{p}(E)$ instead of $S^{2}(E)$ for any $1<p<\infty$.

We need to prove the following result which will be used sometimes in this paper and for which we have not found any reference.

Lemma 2.9. Let $E$ be an operator space and let $n$ be a positive integer.

(a) If $1 \leqslant p<q \leqslant \infty$, then $\|A\|_{S_{n}^{p}(E)} \leqslant n^{1 / p-1 / q}\|A\|_{S_{n}^{q}(E)}$.

(b) If $\|A\|_{S_{n}^{1}(E)}=\sqrt{n}\|A\|_{S_{n}^{2}(E)}$, then $\|A\|_{S_{n}^{1}(E)}=n\|A\|_{S_{n}^{\infty}(E)}$.

Proof. If $p=1$ and $q=\infty$, then (a) follows easily from Corollary 9.8 of [13]. The general case follows by complex interpolation. Let us prove (b). By homogeneity, we assume without loss of generality that $\|A\|_{S_{n}^{2}(E)}=1$. On the other hand, by Theorem 1.5 of $[\mathbf{1 4}]$, we know that

$$
\|A\|_{S_{n}^{2}(E)}=\inf _{A=\alpha B \beta}\|\alpha\|_{S_{n}^{4}}\|B\|_{S_{n}^{\infty}(E)}\|\beta\|_{S_{n}^{4}}=1
$$

where $\alpha, \beta \in M_{n}$ and $B \in M_{n} \otimes E$. Moreover, if $F=\operatorname{span}\left\{A_{i j}: 1 \leqslant i, j \leqslant n\right\}$, we can take $B \in M_{n} \otimes F$. In particular, for all $k \geqslant 1$ there exist $\alpha_{k}, \beta_{k} \in M_{n}$ and $B_{k} \in M_{n} \otimes F$ such that $A=\alpha_{k} B_{k} \beta_{k}, 1 \leqslant\left\|\alpha_{k}\right\|_{S_{n}^{4}}<1+1 / k$ and $\left\|B_{k}\right\|_{S_{n}^{\infty}(F)}=\left\|\beta_{k}\right\|_{S_{n}^{4}}=1$. By the finite dimensionality of $F$, we know that the sequence $\left(\alpha_{k}, B_{k}, \beta_{k}\right)$ belongs to a compact subset of $S_{n}^{4} \times S_{n}^{\infty}(E) \times S_{n}^{4}$. Thus, there exist $\alpha_{0}, \beta_{0} \in M_{n}$ and $B_{0} \in M_{n} \otimes F$ such that $A=\alpha_{0} B_{0} \beta_{0}$ and $\left\|\alpha_{0}\right\|_{S_{n}^{4}}=\left\|B_{0}\right\|_{S_{n}^{\infty}(F)}=\left\|\beta_{0}\right\|_{S_{n}^{4}}=1$. But then, again by Theorem 1.5 of $[\mathbf{1 4}]$,

$$
\left\|\alpha_{0}\right\|_{S_{n}^{2}}\left\|\beta_{0}\right\|_{S_{n}^{2}} \geqslant\|A\|_{S_{n}^{1}(E)}=\sqrt{n} .
$$

Moreover, taking $p=2$ and $q=4$, (a) gives $\left\|\alpha_{0}\right\|_{S_{n}^{2}},\left\|\beta_{0}\right\|_{S_{n}^{2}} \leqslant n^{1 / 4}$. In summary, we get

$$
\left\|\alpha_{0}\right\|_{S_{n}^{2}}=\left\|\beta_{0}\right\|_{S_{n}^{2}}=n^{1 / 4} \quad \text { and } \quad\left\|\alpha_{0}\right\|_{S_{n}^{4}}=\left\|\beta_{0}\right\|_{S_{n}^{4}}=1 .
$$

Then, it is well known that there exists $U, V \in U(n)$ such that $\alpha_{0}=n^{-1 / 4} U$ and $\beta_{0}=n^{-1 / 4} V$. Therefore,

$$
\|A\|_{S_{n}^{\infty}(E)} \leqslant\left\|\alpha_{0}\right\|_{S_{n}^{\infty}}\left\|B_{0}\right\|_{S_{n}^{\infty}(E)}\left\|\beta_{0}\right\|_{S_{n}^{\infty}}=n^{-1 / 2} .
$$

This gives $\|A\|_{S_{n}^{1}(E)} \geqslant n\|A\|_{S_{n}^{\infty}(E)}$, and the reverse inequality follows from (a).

\section{The equivalent notions}

In this section we show the equivalence between some of the notions previously defined. For that purpose we begin by fixing a set of parameters $\left(\Sigma, \boldsymbol{d}_{\Sigma}\right)$. First, we need to prove a technical lemma. 
Lemma 3.1. Let $F$ be an operator space and let $\Gamma$ be a finite subset of $\Sigma$. Let us suppose that, for all $\varepsilon>0$, there exist a family of matrices $X_{\varepsilon}^{\sigma} \in M_{d_{\sigma}} \otimes F$ with $\sigma \in \Gamma$ and such that

(1) $\left(\int_{\Omega}\left\|\sum_{\sigma \in \Gamma} d_{\sigma} \operatorname{tr}\left(X_{\varepsilon}^{\sigma} \zeta^{\sigma}(\omega)\right)\right\|_{F}^{2} \mathrm{~d} \mu(\omega)\right)^{1 / 2} \geqslant \Delta_{\Gamma}-\varepsilon$.

(2) $\left(\sum_{\sigma \in \Gamma} d_{\sigma}\left\|X_{\varepsilon}^{\sigma}\right\|_{S_{d_{\sigma}}^{2}(F)}^{2}\right)^{1 / 2}=\Delta_{\Gamma}^{1 / 2}$

Then

$$
\max _{\sigma \in \Gamma}\left\|X_{\varepsilon}^{\sigma}\right\|_{S_{d_{\sigma}}^{\infty}(F)} \leqslant 1+\xi(\varepsilon)
$$

with $\xi(\varepsilon) \rightarrow 0^{+}$as $\varepsilon \rightarrow 0^{+}$.

Proof. Let $\mathcal{U}$ be an ultrafilter on $\mathbb{R}_{+}$containing all the intervals $(0, \varepsilon)$ with $\varepsilon>0$ and let $F_{\mathcal{U}}$ be the corresponding ultraproduct operator space. Then we define $X^{\sigma}=\left(X_{\varepsilon}^{\sigma}\right)_{\mathcal{U}}$ for $\sigma \in \Gamma$. That is, $X^{\sigma} \in M_{d_{\sigma}} \otimes F_{\mathcal{U}}$. We obviously have

$$
\left(\int_{\Omega}\left\|\sum_{\sigma \in \Gamma} d_{\sigma} \operatorname{tr}\left(X^{\sigma} \zeta^{\sigma}(\omega)\right)\right\|_{F_{\mathcal{U}}}^{2} \mathrm{~d} \mu(\omega)\right)^{1 / 2} \geqslant \Delta_{\Gamma} \quad \text { and }\left(\sum_{\sigma \in \Gamma} d_{\sigma}\left\|X^{\sigma}\right\|_{S_{d_{\sigma}}^{2}\left(F_{\mathcal{U}}\right)}^{2}\right)^{1 / 2}=\Delta_{\Gamma}^{1 / 2} .
$$

By the Hölder inequality and Lemma 2.9, we can write

$$
\begin{aligned}
\Delta_{\Gamma}^{2} & \leqslant \int_{\Omega}\left(\sum_{\sigma \in \Gamma} d_{\sigma}\left\|X^{\sigma}\right\|_{S_{d_{\sigma}}^{1}\left(F_{\mathcal{U}}\right)}\left\|\zeta^{\sigma}(\omega)\right\|_{S_{d_{\sigma}}^{\infty}}\right)^{2} \mathrm{~d} \mu(\omega) \\
& =\left(\sum_{\sigma \in \Gamma} d_{\sigma}\left\|X^{\sigma}\right\|_{S_{d_{\sigma}}^{1}\left(F_{\mathcal{U}}\right)}\right)^{2} \leqslant\left(\sum_{\sigma \in \Gamma} d_{\sigma} \sqrt{d_{\sigma}}\left\|X^{\sigma}\right\|_{S_{d_{\sigma}}^{2}\left(F_{\mathcal{U}}\right)}\right)^{2} \\
& \leqslant \Delta_{\Gamma} \sum_{\sigma \in \Gamma} d_{\sigma}\left\|X^{\sigma}\right\|_{S_{d_{\sigma}}^{2}\left(F_{\mathcal{U}}\right)}^{2}=\Delta_{\Gamma}^{2} .
\end{aligned}
$$

In particular, in this case, Lemma 2.9 and the Hölder inequality are equalities. Therefore, we obtain

$$
\left\|X^{\sigma}\right\|_{S_{d_{\sigma}}^{1}\left(F_{\mathcal{U}}\right)}=\sqrt{d_{\sigma}}\left\|X^{\sigma}\right\|_{S_{d_{\sigma}}^{2}\left(F_{\mathcal{U}}\right)} \quad \text { and } \quad d_{\sigma}=c_{0} \sqrt{d_{\sigma}}\left\|X^{\sigma}\right\|_{S_{d_{\sigma}}^{2}\left(F_{\mathcal{U}}\right)}
$$

for some positive constant $c_{0}$ and any $\sigma \in \Gamma$. Now, Lemma 2.9 gives $\left\|X^{\sigma}\right\|_{S_{d_{\sigma}}^{\infty}\left(F_{\mathcal{U}}\right)}=1 / c_{0}$. But $c_{0}=1$ since

$$
\frac{1}{c_{0}^{2}} \Delta_{\Gamma}=\sum_{\sigma \in \Gamma} d_{\sigma}\left\|X^{\sigma}\right\|_{S_{d_{\sigma}}^{2}\left(F_{\mathcal{U}}\right)}^{2}=\Delta_{\Gamma}
$$

So, we have $\max _{\sigma \in \Gamma}\left\|X^{\sigma}\right\|_{S_{d_{\sigma}}^{\infty}\left(F_{\mathcal{U}}\right)}=1$. Finally, by the isometry $S_{d_{\sigma}}^{\infty}\left(F_{\mathcal{U}}\right)=S_{d_{\sigma}}^{\infty}(F)_{\mathcal{U}}($ see Chapter 5 of [14]) and the definition of ultraproduct operator space, the result follows. 
Theorem 3.2. Let $E$ be an operator space and let us fix a set of parameters $\left(\Sigma, \boldsymbol{d}_{\Sigma}\right)$. The following are then equivalent.

(a) E has $\Sigma$-subtype.

(b) $E$ is $B_{\Sigma}$-convex.

(c) $E$ is uniformly non- $\mathcal{L}^{1}(\Sigma)$.

Proof. Let us suppose that $E$ has $\Sigma$-subtype, we shall see that $E$ is $B_{\Sigma^{-}}$-convex. We know that $\left\|\mathrm{T}_{2}(\Gamma, E)\right\|_{\mathrm{cb}}=(1-\delta) \Delta_{\Gamma}^{1 / 2}$ for some $\Gamma \subset \Sigma$ finite and some $0<\delta \leqslant 1$. Hence, we can write

$$
\begin{aligned}
\frac{1}{\Delta_{\Gamma}} \inf _{B^{\sigma} \text { unitary }}\left\|\sum_{\sigma \in \Gamma} d_{\sigma} \operatorname{tr}\left(A^{\sigma} B^{\sigma}\right)\right\|_{S^{2}(E)} & \leqslant \frac{1}{\Delta_{\Gamma}}\left\|\sum_{\sigma \in \Gamma} d_{\sigma} \operatorname{tr}\left(A^{\sigma} \zeta^{\sigma}\right)\right\|_{S^{2}\left(L_{E}^{2}(\Omega)\right)} \\
& \leqslant \frac{(1-\delta)}{\Delta_{\Gamma}^{1 / 2}}\|A\|_{S^{2}\left(\mathcal{L}_{E}^{2}(\Gamma)\right)} .
\end{aligned}
$$

The result then follows since, by Lemma 2.9, we have

$$
\|A\|_{\mathcal{L}_{S^{2}(E)}^{2}(\Gamma)} \leqslant \Delta_{\Gamma}^{1 / 2} \max _{\sigma \in \Gamma}\left\|A^{\sigma}\right\|_{S_{d_{\sigma}}^{\infty}\left(S^{2}(E)\right)}
$$

Now, to see that $B_{\Sigma}$-convex operator spaces are uniformly non- $\mathcal{L}^{1}(\Sigma)$, we assume that $E$ contains $\mathcal{L}^{1}(\Gamma) \lambda$-uniformly for all $\lambda>1$ and we have to see that $E$ is not $B_{\Sigma^{-} \text {-convex. }}$ We know that, for all $\lambda>1$ and all $\Gamma \subset \Sigma$ finite, there exists a subspace $F_{\Gamma}$ of $S^{2}(E)$ and some isomorphism $\Lambda_{\Gamma}: \mathcal{L}^{1}(\Gamma) \rightarrow F_{\Gamma}$

$$
A \in \mathcal{L}^{1}(\Gamma) \mapsto \sum_{\sigma \in \Gamma} \sum_{i, j=1}^{d_{\sigma}} a_{i j}^{\sigma} x_{i j}^{\sigma} \in F_{\Gamma}, \quad \text { where } A^{\sigma}=\left(a_{i j}^{\sigma}\right),
$$

such that $\left\|\Lambda_{\Gamma}\right\|_{\mathrm{cb}}=1$ and $\left\|\Lambda_{\Gamma}^{-1}\right\| \leqslant \lambda$. On the other hand, if $\sigma \in \Gamma$ and we define the matrix $X^{\sigma}=d_{\sigma}^{-1}\left(x_{i j}^{\sigma}\right)$, we have

$$
\left\|X^{\sigma}\right\|_{S_{d_{\sigma}}^{\infty}\left(S^{2}(E)\right)}=\left\|\operatorname{tr}\left(X^{\sigma} \cdot\right)\right\|_{\mathrm{cb}\left(S_{d_{\sigma}}^{1}, S^{2}(E)\right)} \leqslant\left\|\Lambda_{\Gamma}\right\|_{\mathrm{cb}}=1 .
$$

Hence, by the estimate for the norm of $\Lambda_{\Gamma}^{-1}$ and (3.1), we obtain

$$
\frac{1}{\Delta_{\Gamma}} \inf _{B^{\sigma} \text { unitary }}\left\|\sum_{\sigma \in \Gamma} d_{\sigma} \operatorname{tr}\left(X^{\sigma} B^{\sigma}\right)\right\|_{S^{2}(E)} \geqslant \frac{1}{\lambda} \max _{\sigma \in \Gamma}\left\|X^{\sigma}\right\|_{S_{d_{\sigma}}^{\infty}\left(S^{2}(E)\right)}
$$

since $\|B\|_{\mathcal{L}^{1}(\Gamma)}=\Delta_{\Gamma}$ whenever $B^{\sigma} \in U\left(d_{\sigma}\right)$ for all $\sigma \in \Gamma$. In particular, taking $\lambda \rightarrow 1^{+}$, we conclude that the operator space $E$ is not $B_{\Sigma^{-}}$-convex.

Finally, let us assume that $\left\|\mathrm{T}_{2}(\Gamma, E)\right\|_{\mathrm{cb}}=\Delta_{\Gamma}^{1 / 2}$ for all $\Gamma \subset \Sigma$ finite. We have to see that $E$ contains $\mathcal{L}^{1}(\Gamma) \lambda$-uniformly for all $\lambda>1$. By Lemma 1.7 of [14], we know 
that $\left\|\mathrm{T}_{2}(\Gamma, E)\right\|_{\mathrm{cb}}=\left\|\mathrm{T}_{2}\left(\Gamma, S^{2}(E)\right)\right\|$. In particular, for all $\varepsilon>0$ there exists a family of matrices $X_{\varepsilon}^{\sigma} \in M_{d_{\sigma}} \otimes S^{2}(E)$ such that

$$
\int_{\Omega}\left\|\sum_{\sigma \in \Gamma} d_{\sigma} \operatorname{tr}\left(X_{\varepsilon}^{\sigma} \zeta^{\sigma}(\omega)\right)\right\|_{S^{2}(E)}^{2} \mathrm{~d} \mu(\omega) \geqslant \Delta_{\Gamma}^{2}-\varepsilon \quad \text { and } \sum_{\sigma \in \Gamma} d_{\sigma}\left\|X_{\varepsilon}^{\sigma}\right\|_{S_{d_{\sigma}}^{2}\left(S^{2}(E)\right)}^{2}=\Delta_{\Gamma} .
$$

Moreover, by the Hölder inequality we obtain

$$
\left\|\sum_{\sigma \in \Gamma} d_{\sigma} \operatorname{tr}\left(X_{\varepsilon}^{\sigma} \zeta^{\sigma}(\omega)\right)\right\|_{S^{2}(E)} \leqslant \sum_{\sigma \in \Gamma} d_{\sigma}\left\|X_{\varepsilon}^{\sigma}\right\|_{S_{d_{\sigma}}^{2}\left(S^{2}(E)\right)}\left\|\zeta^{\sigma}(\omega)\right\|_{S_{d_{\sigma}}^{2}} \leqslant \Delta_{\Gamma} .
$$

That is, if we set

$$
f_{\varepsilon}(\omega)=\left\|\sum_{\sigma \in \Gamma} d_{\sigma} \operatorname{tr}\left(X_{\varepsilon}^{\sigma} \zeta^{\sigma}(\omega)\right)\right\|_{S^{2}(E)}^{2}
$$

for $\omega \in \Omega$, then we have

$$
0 \leqslant f_{\varepsilon} \leqslant \Delta_{\Gamma}^{2} \quad \text { and } \quad \Delta_{\Gamma}^{2}-\varepsilon \leqslant \int_{\Omega} f_{\varepsilon}(\omega) \mathrm{d} \mu(\omega) \leqslant \Delta_{\Gamma}^{2} .
$$

In particular, $\mu\left\{\omega \in \Omega: f_{\varepsilon}(\omega)<\Delta_{\Gamma}^{2}-k \varepsilon\right\} \leqslant 1 / k$ for all $k \geqslant 1$. On the other hand, if we fix $U_{0}^{\sigma} \in U\left(d_{\sigma}\right)$ for any $\sigma \in \Gamma$, we define

$$
\mathcal{U}_{0}(\sigma, \delta)=\left\{U^{\sigma} \in U\left(d_{\sigma}\right):\left\|U^{\sigma}-U_{0}^{\sigma}\right\|_{S_{d_{\sigma}}^{2}}<\delta\right\} .
$$

Then we recall that, by the independence of the random matrices $\zeta^{\sigma}$ and their uniform distribution in $U\left(d_{\sigma}\right)$ with respect to the normalized Haar measure $\lambda_{\sigma}$ in $U\left(d_{\sigma}\right)$, we have

$$
\mu\left\{\omega \in \Omega: \zeta^{\sigma}(\omega) \in \mathcal{U}_{0}(\sigma, \delta), \sigma \in \Gamma\right\}=\prod_{\sigma \in \Gamma} \lambda_{\sigma}\left(\mathcal{U}_{0}(\sigma, \delta)\right)>0 .
$$

Therefore, by choosing $k_{0}(\delta)$ such that

$$
k_{0}(\delta)^{-1}<\mu\left\{\omega \in \Omega: \zeta^{\sigma}(\omega) \in \mathcal{U}_{0}(\sigma, \delta), \sigma \in \Gamma\right\},
$$

we obtain the following inequality:

$$
\mu\left\{\omega \in \Omega: f_{\varepsilon}(\omega)<\Delta_{\Gamma}^{2}-k_{0}(\delta) \varepsilon\right\}<\mu\left\{\omega \in \Omega: \zeta^{\sigma}(\omega) \in \mathcal{U}_{0}(\sigma, \delta), \sigma \in \Gamma\right\} .
$$

That is, there exists some $\omega_{0} \in \Omega$ such that $\zeta^{\sigma}\left(\omega_{0}\right) \in \mathcal{U}_{0}(\sigma, \delta)$ for all $\sigma \in \Gamma$ and such that $f_{\varepsilon}\left(\omega_{0}\right) \geqslant \Delta_{\Gamma}^{2}-k_{0}(\delta) \varepsilon$. These two properties give us the following sequence of inequalities:

$$
\begin{aligned}
\sqrt{\Delta_{\Gamma}^{2}-k_{0}(\delta) \varepsilon} & \leqslant\left\|\sum_{\sigma \in \Gamma} d_{\sigma} \operatorname{tr}\left(X_{\varepsilon}^{\sigma}\left[\zeta^{\sigma}\left(\omega_{0}\right)-U_{0}^{\sigma}\right]\right)\right\|_{S^{2}(E)}+\left\|\sum_{\sigma \in \Gamma} d_{\sigma} \operatorname{tr}\left(X_{\varepsilon}^{\sigma} U_{0}^{\sigma}\right)\right\|_{S^{2}(E)} \\
& \leqslant \sum_{\sigma \in \Gamma} d_{\sigma}\left\|X_{\varepsilon}^{\sigma}\right\|_{S_{d_{\sigma}}^{2}\left(S^{2}(E)\right)}\left\|\zeta^{\sigma}\left(\omega_{0}\right)-U_{0}^{\sigma}\right\|_{S_{d_{\sigma}}^{2}}+\left\|\sum_{\sigma \in \Gamma} d_{\sigma} \operatorname{tr}\left(X_{\varepsilon}^{\sigma} U_{0}^{\sigma}\right)\right\|_{S^{2}(E)} \\
& <\delta \Delta_{\Gamma}+\left\|\sum_{\sigma \in \Gamma} d_{\sigma} \operatorname{tr}\left(X_{\varepsilon}^{\sigma} U_{0}^{\sigma}\right)\right\|_{S^{2}(E)}
\end{aligned}
$$


Taking $\varepsilon(\delta)=\delta / k_{0}(\delta)$, it is easy to check that there exists $\gamma_{1}(\delta)>0$ such that

$$
\left\|\sum_{\sigma \in \Gamma} d_{\sigma} \operatorname{tr}\left(X_{\varepsilon(\delta)}^{\sigma} U_{0}^{\sigma}\right)\right\|_{S^{2}(E)} \geqslant \Delta_{\Gamma}-\gamma_{1}(\delta)
$$

and where $\varepsilon(\delta), \gamma_{1}(\delta) \rightarrow 0^{+}$as $\delta \rightarrow 0^{+}$. In particular, since for some other choice of the unitary matrices $U_{0}^{\sigma}(\sigma \in \Gamma)$ we have the same value for $\lambda_{\sigma}\left(\mathcal{U}_{0}(\sigma, \delta)\right)$ (by the translation invariance of the Haar measure $\lambda_{\sigma}$ ), we obtain that $k_{0}(\delta)$ does not depend on the chosen matrices $U_{0}^{\sigma}(\sigma \in \Gamma)$ and (3.2) holds for any family of unitary matrices $U^{\sigma} \in U\left(d_{\sigma}\right)$ with $\sigma \in \Gamma$. Now, given $A \in \mathcal{L}^{1}(\Sigma)$ of norm 1, we use polar decomposition to write $A^{\sigma}=U_{A}^{\sigma}\left|A^{\sigma}\right|$ with $U_{A}^{\sigma} \in U\left(d_{\sigma}\right)$. We then have

$$
\begin{aligned}
\Delta_{\Gamma}-\gamma_{1}(\delta) & \leqslant\left\|\sum_{\sigma \in \Gamma} d_{\sigma} \operatorname{tr}\left(X_{\varepsilon(\delta)}^{\sigma} U_{A}^{\sigma}\left[I-\left|A^{\sigma}\right|\right]\right)\right\|_{S^{2}(E)}+\left\|\sum_{\sigma \in \Gamma} d_{\sigma} \operatorname{tr}\left(X_{\varepsilon(\delta)}^{\sigma} A^{\sigma}\right)\right\|_{S^{2}(E)} \\
& \leqslant \sum_{\sigma \in \Gamma} d_{\sigma}\left\|X_{\varepsilon(\delta)}^{\sigma}\right\|_{S_{d_{\sigma}}^{\infty}\left(S^{2}(E)\right)}\left\|I-\left|A^{\sigma}\right|\right\|_{S_{d_{\sigma}}^{1}}+\left\|\sum_{\sigma \in \Gamma} d_{\sigma} \operatorname{tr}\left(X_{\varepsilon(\delta)}^{\sigma} A^{\sigma}\right)\right\|_{S^{2}(E)} \\
& \leqslant(1+\xi(\delta))\left(\Delta_{\Gamma}-1\right)+\left\|\sum_{\sigma \in \Gamma} d_{\sigma} \operatorname{tr}\left(X_{\varepsilon(\delta)}^{\sigma} A^{\sigma}\right)\right\|_{S^{2}(E)}
\end{aligned}
$$

where the last inequality follows by Lemma 3.1. Let us consider the subspace $F_{\Gamma}$ of $S^{2}(E)$ spanned by the entries of $X_{\varepsilon(\delta)}^{\sigma}$, where $\sigma$ runs over $\Gamma$. The last inequality then gives that the linear isomorphism $\Lambda_{\Gamma}$, given by

$$
A \in \mathcal{L}^{1}(\Gamma) \mapsto \sum_{\sigma \in \Gamma} d_{\sigma} \operatorname{tr}\left(X_{\varepsilon(\delta)}^{\sigma} A^{\sigma}\right) \in F_{\Gamma},
$$

satisfies $\left\|\Lambda_{\Gamma}^{-1}\right\| \leqslant 1+\gamma_{2}(\delta)$ for some $\gamma_{2}(\delta)>0$ satisfying $\gamma_{2}(\delta) \rightarrow 0^{+}$as $\delta \rightarrow 0^{+}$. Moreover, we have that $\left\|\Lambda_{\Gamma}\right\|_{\mathrm{cb}} \leqslant 1+\xi(\delta)$. Namely, given $A \in S^{1}\left(\mathcal{L}^{1}(\Gamma)\right)$, we have

$$
\begin{aligned}
\left\|\sum_{\sigma \in \Gamma} d_{\sigma} \operatorname{tr}\left(X_{\varepsilon(\delta)}^{\sigma} A^{\sigma}\right)\right\|_{S^{1}\left(S^{2}(E)\right)} & \leqslant \sum_{\sigma \in \Gamma} d_{\sigma}\left\|X_{\varepsilon(\delta)}^{\sigma}\right\|_{S_{d_{\sigma}}^{\infty}\left(S^{2}(E)\right)}\left\|A^{\sigma}\right\|_{S_{d_{\sigma}}^{1}\left(S^{1}\right)} \\
& \leqslant(1+\xi(\delta))\|A\|_{S^{1}\left(\mathcal{L}^{1}(\Gamma)\right)} .
\end{aligned}
$$

The first inequality follows by an inequality of Hölder type (see, for example, Lemma 3.3 of $[\mathbf{3}])$. That is, we have seen that $\left\|\Lambda_{\Gamma}\right\|_{\mathrm{cb}}=\left\|\Lambda_{\Gamma} \otimes I_{S^{1}}\right\| \leqslant 1+\xi(\delta)$. Therefore, $\left\|\Lambda_{\Gamma}\right\|_{\mathrm{cb}}\left\|\Lambda_{\Gamma}^{-1}\right\| \leqslant 1+\gamma_{3}(\delta)$ with $\gamma_{3}(\delta) \rightarrow 0^{+}$as $\delta \rightarrow 0^{+}$. Therefore, taking $\delta \rightarrow 0^{+}$we obtain that $E$ contains $\mathcal{L}^{1}(\Gamma) \lambda$-uniformly for all $\lambda>1$, as we wanted. This completes the proof.

Remark 3.3. As in Lemma 3.1, we could have used an argument with ultraproducts to show that uniformly non- $\mathcal{L}^{1}(\Sigma)$ operator spaces have $\Sigma$-subtype. This alternative proof is a bit shorter. However, for the sake of clarity, we have preferred to give the more explicit argument used in the proof of Theorem 3.2. 
As was pointed out in Remark 2.8, it is very natural to wonder whether Definition 2.5 is affected if we change $S^{2}(E)$ to $S^{p}(E)$ with $1<p<\infty$. The notion of $B_{\Sigma}$-convexity should not depend on the choice of the exponent $p$ and, fortunately, this is the case.

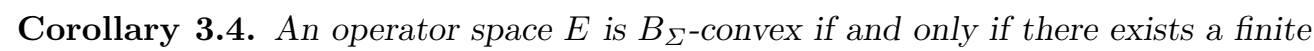
subset $\Gamma$ of $\Sigma$ such that

$$
\frac{1}{\Delta_{\Gamma}} \inf _{B^{\sigma} \text { unitary }}\left\|\sum_{\sigma \in \Gamma} d_{\sigma} \operatorname{tr}\left(A^{\sigma} B^{\sigma}\right)\right\|_{S^{p}(E)} \leqslant(1-\delta) \max _{\sigma \in \Gamma}\left\|A^{\sigma}\right\|_{S_{d_{\sigma}}^{\infty}\left(S^{p}(E)\right)}
$$

for some $1<p<\infty$ and any family $\left\{A^{\sigma} \in M_{d_{\sigma}} \otimes S^{p}(E)\right\}_{\sigma \in \Gamma}$.

Proof. By Lemma 1.7 of [14] and Theorem 3.2, we know that $E$ is $B_{\Sigma}$-convex if and only if there exists a finite subset $\Gamma$ of $\Sigma$ such that $\left\|\mathrm{T}_{2}\left(\Gamma, S^{2}(E)\right)\right\|<\Delta_{\Gamma}^{1 / 2}$. On the other hand, given $1<p, q<\infty$, we claim that

$$
\left\|\mathrm{T}_{2}\left(\Gamma, S^{p}(E)\right)\right\|<\Delta_{\Gamma}^{1 / 2} \Longleftrightarrow\left\|\mathrm{T}_{2}\left(\Gamma, S^{q}(E)\right)\right\|<\Delta_{\Gamma}^{1 / 2} .
$$

By the Hölder inequality we have $\left\|\mathrm{T}_{2}(\Gamma, F)\right\| \leqslant \Delta_{\Gamma}^{1 / 2}$ for any operator space $F$. Then (3.3) follows by complex interpolation with $S^{1}(E)$ and $S^{\infty}(E)$. In particular, given $1<$

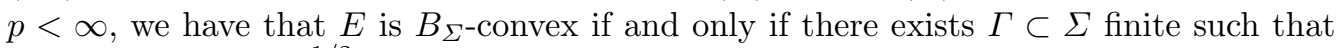
$\left\|\mathrm{T}_{2}\left(\Gamma, S^{p}(E)\right)\right\|<\Delta_{\Gamma}^{1 / 2}$. But, proceeding as in the proof of Theorem 3.2, we can see that the desired inequality is equivalent to this last condition. This completes the proof.

Remark 3.5. By similar arguments, if $1<p<\infty$, we can also replace $S^{2}(E)$ by $S^{p}(E)$ in the definition of uniformly non- $\mathcal{L}^{1}(\Sigma)$ operator spaces.

\section{Tensor submultiplicativity}

As we pointed out in Remark 2.2, it seems that the classical set of parameters and those $\Sigma$ having $\boldsymbol{d}_{\Sigma}$ unbounded are the most relevant ones. In this section we shall see that,

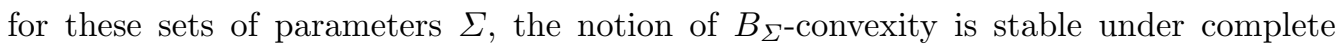
isomorphy and the notion of containing $\mathcal{L}^{1}(\Gamma) \lambda$-uniformly does not depend on $\lambda>1$. The commutative analogues of these results are very well known (see, for example, [5] or $[\mathbf{1 1}])$. In order to prove these results, we need to fix some notation. Let $\left(\Sigma, \boldsymbol{d}_{\Sigma}\right)$ be a set of parameters. Given two subsets $\Gamma^{1}$ and $\Gamma^{2}$ of $\Sigma$, we define their tensor product as

$$
\Gamma^{1} \otimes \Gamma^{2}=\left\{\sigma_{1} \otimes \sigma_{2}: \sigma_{j} \in \Gamma^{j}, j=1,2\right\}, \quad \text { where } d_{\sigma_{1} \otimes \sigma_{2}}=d_{\sigma_{1}} d_{\sigma_{2}} .
$$

We say that $\left(\Sigma, \boldsymbol{d}_{\Sigma}\right)$ is $\otimes$-closed if, for any pair of subsets $\Gamma^{1}$ and $\Gamma^{2}$ of $\Sigma$, there exists an injective mapping $j: \Gamma^{1} \otimes \Gamma^{2} \rightarrow \Sigma$ such that $d_{j\left(\sigma_{1} \otimes \sigma_{2}\right)}=d_{\sigma_{1} \otimes \sigma_{2}}$ for all $\sigma_{1} \otimes \sigma_{2} \in \Gamma^{1} \otimes \Gamma^{2}$. Also, given $\Gamma \subset \Sigma$ finite, we define

$$
\mathrm{N}_{\Gamma}(E)=\frac{1}{\sqrt{\Delta_{\Gamma}}}\left\|\mathrm{T}_{2}(\Gamma, E)\right\|_{\mathrm{cb}}
$$

Lemma 4.1. If $\left(\Sigma, \boldsymbol{d}_{\Sigma}\right)$ is $\otimes$-closed, then $\mathrm{N}_{\Gamma^{1} \otimes \Gamma^{2}}(E) \leqslant \mathrm{N}_{\Gamma^{1}}(E) \mathrm{N}_{\Gamma^{2}}(E)$ for any pair of finite subsets $\Gamma^{1}$ and $\Gamma^{2}$ of $\Sigma$. 
Proof. Let us consider a family

$$
A=\left\{A^{\sigma_{1} \otimes \sigma_{2}} \in M_{d_{\sigma_{1}} d_{\sigma_{2}}} \otimes S^{2}(E): \sigma_{1} \in \Gamma^{1}, \sigma_{2} \in \Gamma^{2}\right\} .
$$

Let

$$
A^{\sigma_{1} \otimes \sigma_{2}}(\omega)=\zeta^{j\left(\sigma_{1} \otimes \sigma_{2}\right)}(\omega) A^{\sigma_{1} \otimes \sigma_{2}}
$$

for $\omega \in \Omega$. Since $\zeta^{j\left(\sigma_{1} \otimes \sigma_{2}\right)}$ is uniformly distributed on the unitary group $U\left(d_{\sigma_{1}} d_{\sigma_{2}}\right)$ and $\zeta^{\sigma_{1}}\left(\omega_{1}\right) \otimes \zeta^{\sigma_{2}}\left(\omega_{2}\right)$ is unitary, we have

$$
\begin{aligned}
\int_{\Omega}\left\|\sum_{\sigma_{j} \in \Gamma^{j}} d_{\sigma_{1}} d_{\sigma_{2}} \operatorname{tr}\left(A^{\sigma_{1} \otimes \sigma_{2}}(\omega)\right)\right\|_{S^{2}(E)}^{2} \mathrm{~d} \mu(\omega) \\
\quad=\int_{\Omega}\left\|\sum_{\sigma_{j} \in \Gamma^{j}} d_{\sigma_{1}} d_{\sigma_{2}} \operatorname{tr}\left(A^{\sigma_{1} \otimes \sigma_{2}}(\omega)\left(\zeta^{\sigma_{1}}\left(\omega_{1}\right) \otimes \zeta^{\sigma_{2}}\left(\omega_{2}\right)\right)\right)\right\|_{S^{2}(E)}^{2} \mathrm{~d} \mu(\omega)
\end{aligned}
$$

for all $\omega_{1}, \omega_{2} \in \Omega$. Therefore, if we write

$$
X^{\sigma_{1}}\left(\omega, \omega_{2}\right)=\sum_{\sigma_{2} \in \Gamma^{2}} d_{\sigma_{2}} \operatorname{tr}\left(A^{\sigma_{1} \otimes \sigma_{2}}(\omega) \zeta^{\sigma_{2}}\left(\omega_{2}\right)\right) \in M_{d_{\sigma_{1}}} \otimes S^{2}(E),
$$

we obtain the following estimate:

$$
\begin{aligned}
& \int_{\Omega}\left\|\sum_{\sigma_{j} \in \Gamma^{j}} d_{\sigma_{1}} d_{\sigma_{2}} \operatorname{tr}\left(A^{\sigma_{1} \otimes \sigma_{2}}(\omega)\right)\right\|_{S^{2}(E)}^{2} \mathrm{~d} \mu(\omega) \\
& =\int_{\Omega \times \Omega \times \Omega}\left\|\sum_{\sigma_{j} \in \Gamma^{j}} d_{\sigma_{1}} d_{\sigma_{2}} \operatorname{tr}\left(A^{\sigma_{1} \otimes \sigma_{2}}(\omega)\left(\zeta^{\sigma_{1}}\left(\omega_{1}\right) \otimes \zeta^{\sigma_{2}}\left(\omega_{2}\right)\right)\right)\right\|_{S^{2}(E)}^{2} \mathrm{~d} \mu\left(\omega_{1}\right) \mathrm{d} \mu\left(\omega_{2}\right) \mathrm{d} \mu(\omega) \\
& =\int_{\Omega \times \Omega \times \Omega}\left\|\sum_{\sigma_{1} \in \Gamma^{1}} d_{\sigma_{1}} \operatorname{tr}\left(X^{\sigma_{1}}\left(\omega, \omega_{2}\right) \zeta^{\sigma_{1}}\left(\omega_{1}\right)\right)\right\|_{S^{2}(E)}^{2} \mathrm{~d} \mu\left(\omega_{1}\right) \mathrm{d} \mu\left(\omega_{2}\right) \mathrm{d} \mu(\omega) \\
& \leqslant \mathrm{N}_{\Gamma^{1}}(E)^{2} \Delta_{\Gamma^{1}} \sum_{\sigma_{1} \in \Gamma^{1}} d_{\sigma_{1}} \int_{\Omega \times \Omega}\left\|X^{\sigma_{1}}\left(\omega, \omega_{2}\right)\right\|_{S_{d_{\sigma_{1}}}^{2}\left(S^{2}(E)\right)}^{2} \mathrm{~d} \mu\left(\omega_{2}\right) \mathrm{d} \mu(\omega) \\
& \leqslant \mathrm{N}_{\Gamma^{1}}(E)^{2} \mathrm{~N}_{\Gamma^{2}}(E)^{2} \Delta_{\Gamma^{1}} \Delta_{\Gamma^{2}} \sum_{\sigma_{j} \in \Gamma^{j}} d_{\sigma_{1}} d_{\sigma_{2}} \int_{\Omega}\left\|A^{\sigma_{1} \otimes \sigma_{2}}(\omega)\right\|_{S_{d_{\sigma_{1}} d_{\sigma_{2}}}^{2}\left(S^{2}(E)\right)}^{2} \mathrm{~d} \mu(\omega) \\
& =\mathrm{N}_{\Gamma^{1}}(E)^{2} \mathrm{~N}_{\Gamma^{2}}(E)^{2} \Delta_{\Gamma^{1}} \Delta_{\Gamma^{2}} \sum_{\sigma_{j} \in \Gamma^{j}} d_{\sigma_{1}} d_{\sigma_{2}}\left\|A^{\sigma_{1} \otimes \sigma_{2}}\right\|_{S_{d_{\sigma_{1}} d_{\sigma_{2}}}^{2}\left(S^{2}(E)\right)}^{2} .
\end{aligned}
$$

The last equality follows by the unitarity of $\zeta^{j\left(\sigma_{1} \otimes \sigma_{2}\right)}(\omega)$. Now, since $\Delta_{\Gamma^{1} \otimes \Gamma^{2}}=\Delta_{\Gamma^{1}} \Delta_{\Gamma^{2}}$ we obtain the desired inequality. This completes the proof.

Proposition 4.2. If $\left(\Sigma, \boldsymbol{d}_{\Sigma}\right)$ is $\otimes$-closed, then $B_{\Sigma}$-convexity is stable under complete isomorphism. 
Proof. Let us assume that $E$ is $B_{\Sigma}$-convex and let $F$ be an operator space completely isomorphic to $E$. By Theorem 3.2, we know that there exists some $\Gamma_{0} \subset \Sigma$ finite such that $\mathrm{N}_{\Gamma_{0}}(E)<1$ and it suffices to see that there exists some $\Gamma \subset \Sigma$ finite such that $\mathrm{N}_{\Gamma}(F)<1$. However, if $d_{\mathrm{cb}}(E, F)$ stands for the cb-distance between $E$ and $F$, we have

$$
\mathrm{N}_{\Gamma_{0}^{\otimes n}}(F) \leqslant d_{\mathrm{cb}}(E, F) \mathrm{N}_{\Gamma_{0}^{\otimes n}}(E),
$$

where $n$ is any positive integer and $\Gamma_{0}^{\otimes n}=\Gamma_{0} \otimes \Gamma_{0} \otimes \cdots \otimes \Gamma_{0}$ with $n$ factors. Now, by Lemma 4.1, we know that $\mathrm{N}_{\Gamma_{0}^{\otimes n}}(E) \leqslant \mathrm{N}_{\Gamma_{0}}(E)^{n}$. Finally, we are done by taking $\Gamma=\Gamma_{0}^{\otimes n}$ with $n$ large enough. This completes the proof.

Remark 4.3. Proposition 4.2 obviously holds for the classical set of parameters. On the other hand, if we consider the set of parameters given by $\Sigma=\mathbb{N} \times \mathbb{N}$ and $d_{\sigma_{j k}}=2^{k}$, then $\sigma_{11}$ generates the whole set of parameters by taking tensor powers of it. This set satisfies that $\boldsymbol{d}_{\Sigma}$ is unbounded and is again $\otimes$-closed. Therefore, Proposition 4.2 also holds for it. Moreover, in $\S 6$ we shall prove that the notion of $B_{\Sigma}$-convexity does not depend on $\Sigma$ whenever we work with sets of parameters with $\boldsymbol{d}_{\Sigma}$ unbounded. In particular, we have seen that Proposition 4.2 holds for any set of parameters with $\boldsymbol{d}_{\Sigma}$ unbounded.

Proposition 4.4. Let us suppose that $\left(\Sigma, \boldsymbol{d}_{\Sigma}\right)$ is $\otimes$-closed and let $E$ be an operator space containing $\mathcal{L}^{1}(\Gamma) \lambda$-uniformly for some $\lambda>1$. Then, for all $\tau>1$, E contains $\mathcal{L}^{1}(\Gamma) \tau$-uniformly.

Proof. We have already seen in the proof of Theorem 3.2 that, if $E$ contains $\mathcal{L}^{1}(\Gamma)$ $\lambda$-uniformly, we obtain

$$
\frac{1}{\Delta_{\Gamma}} \inf _{B^{\sigma} \text { unitary }}\left\|\sum_{\sigma \in \Gamma} d_{\sigma} \operatorname{tr}\left(X^{\sigma} B^{\sigma}\right)\right\|_{S^{2}(E)} \geqslant \frac{1}{\lambda} \max _{\sigma \in \Gamma}\left\|X^{\sigma}\right\|_{S_{d_{\sigma}}^{\infty}\left(S^{2}(E)\right)}
$$

for all $\Gamma \subset \Sigma$ finite and a certain family of matrices $X^{\sigma} \in M_{d_{\sigma}} \otimes S^{2}(E)$, with $\sigma \in \Gamma$. On the other hand, from this inequality it is not difficult to see using Lemma 2.9 that

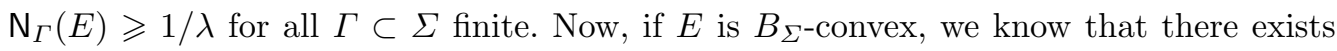
some finite subset $\Gamma_{0}$ of $\Sigma$ such that $\mathrm{N}_{\Gamma_{0}}(E)<1$. Therefore, by Lemma 4.1 , we would have

$$
\frac{1}{\lambda} \leqslant \mathrm{N}_{\Gamma_{0}^{\otimes n}}(E) \leqslant \mathrm{N}_{\Gamma_{0}}(E)^{n} \rightarrow 0^{+} \quad \text { as } n \rightarrow \infty
$$

This gives that $E$ is not $B_{\Sigma}$-convex. But, by Theorem 3.2, this is equivalent to saying that $E$ contains $\mathcal{L}^{1}(\Gamma) \tau$-uniformly for all $\tau>1$. This completes the proof.

Remark 4.5. Proposition 4.4 obviously holds for the classical set of parameters. Moreover, by arguments similar to the ones given in Remark 4.3, Proposition 4.4 also holds for any set of parameters $\left(\Sigma, \boldsymbol{d}_{\Sigma}\right)$ with $\boldsymbol{d}_{\Sigma}$ unbounded. 


\section{Non-trivial $\Sigma$-type and $B_{\Sigma^{-}}$-convexity}

In this section, $\Sigma_{0}$ will stand for the classical set of parameters. We begin by showing that any operator space having non-trivial $\Sigma$-type is automatically $B_{\Sigma}$-convex. However, the most interesting point is that, in contrast with the classical theory, the converse is false. We shall provide examples of $B_{\Sigma_{0}}$-convex operator spaces failing to have $\Sigma_{0}$-type $p$ for any $1<p \leqslant 2$. This is a very important difference between the commutative and non-commutative contexts. Namely, it turns out that we cannot expect to obtain an operator space version of the Maurey-Pisier Theorem. We recall that this result asserts that, for any infinite-dimensional Banach space $B$, the supremum of those $p \in[1,2]$ for which $B$ has type $p$ coincides with the minimum of those $1 \leqslant q \leqslant 2$ for which $B$ contains $l_{n}^{q}$ uniformly (see [9] for more details on this topic).

The examples we are giving are the well-known row and column operator spaces $R$ and $C$ (see, for example, $[\mathbf{1 3}]$ for the definition of these spaces). This is even more surprising since $R$ and $C$ are Hilbertian. Moreover, we shall provide some other examples of Hilbertian operator spaces having sharp $\Sigma_{0}$-type $p$ for any $1<p \leqslant 2$, which are obviously $B_{\Sigma_{0}}$-convex. Finally, we shall use a result of Pisier to show that $\min l^{2}$ and $\max l^{2}$ are Hilbertian operator spaces failing the $B_{\Sigma_{0}}$-convexity.

Proposition 5.1. If $E$ has non-trivial $\Sigma$-type, then $E$ is $B_{\Sigma}$-convex.

Proof. Let $\Gamma$ be any finite subset of $\Sigma$ and let us suppose that $E$ has $\Sigma$-type $p$ for some $1<p \leqslant 2$. By the operator space version of the classical Minkowski inequality (see, for example, $[\mathbf{3}])$, we have that the natural mapping

$$
\mathcal{L}_{S^{2}(E)}^{p}(\Gamma) \rightarrow S^{2}\left(\mathcal{L}_{E}^{p}(\Gamma)\right)
$$

is completely contractive. In particular, if $\mathcal{K}_{p}(E, \Sigma)$ stands for the cb-norm of $\mathrm{T}_{p}(E)$, we have $\left\|\mathrm{T}_{p}\left(\Gamma, S^{2}(E)\right)\right\| \leqslant \mathcal{K}_{p}(E, \Sigma)$. Hence,

$$
\left\|\mathrm{T}_{2}(\Gamma, E)\right\|_{\mathrm{cb}}=\left\|\mathrm{T}_{2}\left(\Gamma, S^{2}(E)\right)\right\| \leqslant\left\|\mathrm{T}_{p}\left(\Gamma, S^{2}(E)\right)\right\| \Delta_{\Gamma}^{1 / p-1 / 2} \leqslant \mathcal{K}_{p}(E, \Sigma) \Delta_{\Gamma}^{1 / p-1 / 2},
$$

where the first inequality follows easily from Lemma 2.9. Therefore, taking $\Gamma$ large enough that $\mathcal{K}_{p}(E, \Sigma)<\Delta_{\Gamma}^{1-1 / p}$, we conclude that $E$ has $\Sigma$-subtype. Thus, $E$ is $B_{\Sigma^{-c o n v e x ~ b y ~}}$ Theorem 3.2. This completes the proof.

The following result has its origins in an unpublished result of Magdalena Musat which asserts that $S^{2}(R)$ and $S^{2}(C)$ are super-reflexive Banach spaces. After some conversations, initiated by Marius Junge and Gilles Pisier, Timur Oikhberg found a surprisingly simple proof of this fact. The next theorem is based on the techniques employed there. First, we fix some notation. As usual, given $0<\theta<1$, we shall denote by $R(\theta)$ the complex interpolation operator space $(R, C)_{\theta}$. Analogously, $C(\theta)$ stands for $(C, R)_{\theta}=R(1-\theta)$. By convention, we also set $R(0)=C(1)=R$ and $C(0)=R(1)=C$.

Theorem 5.2. Let $1 \leqslant p \leqslant 2$, then $R(1 / p)$ and $C(1 / p)$ are $B_{\Sigma_{0}}$-convex Hilbertian operator spaces having $\Sigma_{0}$-type $p$. Moreover, if $1 \leqslant p<2$, then $R(1 / p)$ and $C(1 / p)$ do not have $\Sigma_{0}$-type $q$ for any $p<q \leqslant 2$. 
Proof. Let us suppose that $R(1 / p)$ and $C(1 / p)$ are not $B_{\Sigma_{0}}$-convex. Then, by Theorem 3.2 and Remark 2.7, the spaces $S^{2}(R(1 / p))$ and $S^{2}(C(1 / p))$ should not have type greater than 1 in the Banach space sense. However, we claim that both spaces have type $\frac{4}{3}$. Therefore, $R(1 / p)$ and $C(1 / p)$ are $B_{\Sigma_{0}}$-convex. To prove our claim we recall that, by Theorem 1.1 of [14], we have

$$
S^{2}(R)=R\left(\frac{1}{2}\right) \otimes_{h} R \otimes_{h} R\left(\frac{1}{2}\right) \quad \text { and } \quad S^{2}(C)=C\left(\frac{1}{2}\right) \otimes_{h} C \otimes_{h} C\left(\frac{1}{2}\right)
$$

completely isometrically. Now, since the Haagerup tensor product commutes with the complex interpolation functor, we can write

$$
\left.\begin{array}{l}
S^{2}(R)=\left(R \otimes_{h} R \otimes_{h} R, C \otimes_{h} R \otimes_{h} C\right)_{1 / 2}, \\
S^{2}(C)=\left(C \otimes_{h} C \otimes_{h} C, R \otimes_{h} C \otimes_{h} R\right)_{1 / 2}
\end{array}\right\}
$$

completely isometrically. But, as Banach spaces, $R \otimes_{h} R \otimes_{h} R$ and $C \otimes_{h} C \otimes_{h} C$ are isometrically isomorphic to a Hilbert space. In particular, (5.1) gives that $S^{2}(R)$ and $S^{2}(C)$ have type $\frac{4}{3}$ in the Banach space sense. Hence, by complex interpolation, the same happens with $S^{2}(R(1 / p))$ and $S^{2}(C(1 / p))$.

On the other hand, by the reiteration theorem for the complex interpolation method, we have

$$
R(1 / p)=\left(R\left(\frac{1}{2}\right), C\right)_{(2 / p)-1} \quad \text { and } \quad C(1 / p)=\left(C\left(\frac{1}{2}\right), R\right)_{(2 / p)-1} .
$$

But $R\left(\frac{1}{2}\right)=C\left(\frac{1}{2}\right)$ is an $\mathrm{OH}$ operator space (see $[\mathbf{1 3}]$ ). Therefore, it is easy to check that $R\left(\frac{1}{2}\right)$ has $\Sigma_{0}$-type 2 (see $[\mathbf{2}]$ for more on this topic). In particular, by complex interpolation, we get that $R(1 / p)$ and $C(1 / p)$ have at least $\Sigma_{0}$-type $q$, where

$$
\frac{1}{q}=\frac{1-(2 / p-1)}{2}+\frac{2 / p-1}{1}=\frac{1}{p} .
$$

That is, $R(1 / p)$ and $C(1 / p)$ have $\Sigma_{0}$-type $p$. Now let $p<q \leqslant 2$, we want to see that $R(1 / p)$ and $C(1 / p)$ do not have $\Sigma_{0}$-type $q$. Following the notation introduced in Remark 2.4 , we obviously have that

$$
\left\|\mathrm{T}_{q}\left(\Sigma_{0}, E\right)\right\|_{\mathrm{cb}} \geqslant\left\|\mathrm{T}_{q}^{q}\left(\Sigma_{0}, E\right)\right\|_{\mathrm{cb}}=\left\|\mathrm{T}_{q}^{q}\left(\Sigma_{0}, S^{q}(E)\right)\right\|
$$

for any operator space $E$. Hence, we just need to check that $\mathrm{T}_{q}^{q}\left(\Sigma_{0}, S^{q}(E)\right)$ is not bounded if $E=R(1 / p)$ or $E=C(1 / p)$. But, by the Khintchine-Kahane inequalities, this is equivalent to saying that $S^{q}(R(1 / p))$ and $S^{q}(C(1 / p))$ do not have type $q$ in the Banach space sense for any $p<q \leqslant 2$. Let us recall that

$$
\begin{aligned}
S^{q}(R(1 / p)) & =\left(S^{\infty}(R(1 / p)), S^{1}(R(1 / p))\right)_{1 / q} \\
& =\left(C \otimes_{h} R(1 / p) \otimes_{h} R, R \otimes_{h} R(1 / p) \otimes_{h} C\right)_{1 / q} \\
& =C(1 / q) \otimes_{h} R(1 / p) \otimes_{h} R(1 / q) .
\end{aligned}
$$

Analogously, we obtain $S^{q}(C(1 / p))=C(1 / q) \otimes_{h} C(1 / p) \otimes_{h} R(1 / q)$. Let us consider the subspace of $S^{q}(R(1 / p))$ corresponding to $C(1 / q) \otimes_{h} R(1 / p)$. We can then write

$$
\begin{aligned}
C(1 / q) \otimes_{h} R(1 / p) & =\left(C \otimes_{h} R(1 / p), R \otimes_{h} R(1 / p)\right)_{1 / q} \\
& =\left(\left(C \otimes_{h} R, C \otimes_{h} C\right)_{1 / p},\left(R \otimes_{h} R, R \otimes_{h} C\right)_{1 / p}\right)_{1 / q}
\end{aligned}
$$


completely isometrically. But, as Banach spaces, $R \otimes_{h} R$ and $C \otimes_{h} C$ are isometrically isomorphic to $S^{2}$. Therefore, we have

$$
C(1 / q) \otimes_{h} R(1 / p)=\left(\left(S^{\infty}, S^{2}\right)_{1 / p},\left(S^{2}, S^{1}\right)_{1 / p}\right)_{1 / q}=\left(S^{2 p}, S^{2 p / p+1}\right)_{1 / q}=S^{2 p q / p+q}
$$

isometrically. Finally, since $2 p q<q(p+q)$ whenever $p<q$, we have that $C(1 / q) \otimes_{h} R(1 / p)$ cannot have type $q$ in the Banach space sense. Consequently, the same happens for $S^{q}(R(1 / p))$. A similar argument gives that $S^{q}(C(1 / p))$ cannot have type $q$ in the Banach space sense. This completes the proof.

Remark 5.3. In particular, by Theorem 5.2, the row and column operator spaces are examples of $B_{\Sigma_{0}}$-convex Hilbertian operator spaces failing to have non-trivial $\Sigma_{0}$-type.

Remark 5.4. Although we give more details in $\S 6$, it is a simple consequence of Theorem 3.2 that $B_{\Sigma_{0}}$-convexity is the strongest condition among the possible sets of parameters we are working with. That is, a $B_{\Sigma_{0}}$-convex operator space is automatically $B_{\Sigma}$-convex for any other set of parameters $\Sigma$. In particular, the examples treated in

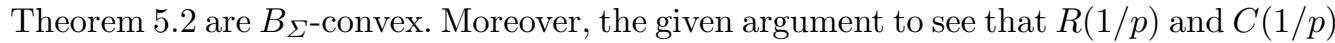
have $\Sigma_{0}$-type $p$ for any $1 \leqslant p \leqslant 2$ remains valid for any other set of parameters $\Sigma$.

Once we have found examples of $B_{\Sigma_{0}}$-convex Hilbertian operator spaces having sharp $\Sigma_{0}$-type $p$ for any $1 \leqslant p \leqslant 2$, we now show that $\min l^{2}$ and $\max l^{2}$ are Hilbertian operator spaces failing to be $B_{\Sigma_{0}}$-convex. This is based on Example 4.2 of [14], where Pisier makes the following construction. Let $M_{2}$ be the algebra of $2 \times 2$ complex-valued matrices equipped with its normalized trace $t$ and let us set $\left(A_{k}, t_{k}\right)=\left(M_{2}, t\right)$ for any $k \geqslant 1$. We then consider the so-called hyperfinite $\mathrm{II}_{1}$ factor

$$
(\mathcal{M}, \tau)=\bigotimes_{k=1}^{\infty}\left(A_{k}, t_{k}\right)
$$

Let $\mathcal{M}_{n}$ stand for the subalgebra of $\mathcal{M}$ corresponding to $A_{1} \otimes \cdots \otimes A_{n}$. Let us consider the element of $\mathcal{M} \otimes \min l^{2}$ given by $d_{n}=V_{n} \otimes e_{n}$, where we write $\left(e_{i}\right)$ for the canonical basis of $l^{2}$ and $\left(V_{n}\right)$ is a sequence in $\mathcal{M}$ satisfying $V_{n} \in \mathcal{M}_{n}$ for all $n \geqslant 1, E^{\mathcal{M}_{n}}\left(V_{n+1}\right)=0$ and the canonical anti-commutation relations

$$
V_{i} V_{j}^{\star}+V_{j}^{\star} V_{i}=\delta_{i j} I \quad \text { and } \quad V_{i} V_{j}+V_{j} V_{i}=0 .
$$

Then, from these properties, Pisier shows that for all finite sequence of scalars $\left(\alpha_{k}\right)$ with $1 \leqslant k \leqslant n$ and for all $1 \leqslant p \leqslant \infty$ we have

$$
\frac{1}{2} \sup _{1 \leqslant k \leqslant n}\left|\alpha_{k}\right| \leqslant\left\|\sum_{k=1}^{n} \alpha_{k} d_{k}\right\|_{L^{p}\left(\mathcal{M} ; \min l^{2}\right)} \leqslant \sup _{1 \leqslant k \leqslant n}\left|\alpha_{k}\right|,
$$

where $L^{p}\left(\mathcal{M} ; \min l^{2}\right)$ denotes the non-commutative $L^{p}$ space defined in $(\mathcal{M}, \tau)$ and with values in $\min l^{2}$. In particular, inequalities (5.2) tell us that the Banach-Mazur distance between some subspace of $L^{p}\left(\mathcal{M}_{n} ; \min l^{2}\right)$ and $l_{n}^{\infty}$ is bounded above by 2 for all $n \geqslant 1$ and all $1 \leqslant p \leqslant \infty$. Then, recalling the natural embedding of $l_{n}^{1}$ into $l_{2^{n}}^{\infty}$ and taking $p=2$ in $(5.2)$, it is easy to see that $S^{2}\left(\min l^{2}\right)$ contains $l_{n}^{1}$ uniformly in the Banach space sense. Therefore, by Remark 2.7 and Theorem 3.2, we have that $\min l^{2}$ is not $B_{\Sigma_{0}}$-convex. By a duality argument, the same happens for $\max l^{2}$. 


\section{On the independence with respect to $\Sigma$}

In this last section we study the dependence of the notion of $B_{\Sigma}$-convexity with respect to the set of parameters $\Sigma$. We begin by showing the independence with respect to $\Sigma$ when we work with sets of parameters satisfying that $\boldsymbol{d}_{\Sigma}$ is an unbounded family. After that, we shall give two interesting equivalent formulations of the possible independence of $B_{\Sigma}$-convexity with respect to any set of parameters $\Sigma$.

Proposition 6.1. Let us consider two sets of parameters $\left(\Sigma_{1}, \boldsymbol{d}_{\Sigma_{1}}\right)$ and $\left(\Sigma_{2}, \boldsymbol{d}_{\Sigma_{2}}\right)$ with $\boldsymbol{d}_{\Sigma_{1}}$ and $\boldsymbol{d}_{\Sigma_{2}}$ unbounded. Let $E$ be an operator space, then $E$ is $B_{\Sigma_{1}}$-convex if and only if $E$ is $B_{\Sigma_{2}}$-convex.

Proof. By Theorem 3.2, we just need to check that $E$ contains $\mathcal{L}^{1}(\Gamma) \lambda$-uniformly $\left(\Gamma \subset \Sigma_{1}\right)$ for all $\lambda>1$ if and only if $E$ contains $\mathcal{L}^{1}(\Gamma) \lambda$-uniformly $\left(\Gamma \subset \Sigma_{2}\right.$ ) for all $\lambda>1$. In particular, it suffices to see that $\mathcal{L}^{1}\left(\Sigma_{1}\right)$ contains $\mathcal{L}^{1}(\Gamma) \lambda$-uniformly $\left(\Gamma \subset \Sigma_{2}\right)$ for all $\lambda>1$ and that $\mathcal{L}^{1}\left(\Sigma_{2}\right)$ contains $\mathcal{L}^{1}(\Gamma) \lambda$-uniformly $\left(\Gamma \subset \Sigma_{1}\right)$ for all $\lambda>1$. But this follows from the unboundedness of $\boldsymbol{d}_{\Sigma_{1}}$ and $\boldsymbol{d}_{\Sigma_{2}}$. Namely, given $\Gamma \subset \Sigma_{2}$ finite, we know that there exists $\Lambda \subset \Sigma_{1}$ and a bijection $\tau: \Gamma \rightarrow \Lambda$ such that $d_{\sigma} \leqslant d_{\tau(\sigma)}$ for all $\sigma \in \Gamma$. In particular we can consider the linear mapping $\mathrm{S}_{\Gamma}: \mathcal{L}^{1}(\Gamma) \rightarrow \mathcal{L}^{1}(\Lambda)$ given by

$$
\mathrm{S}_{\Gamma}(A)_{i j}^{\tau(\sigma)}=\frac{d_{\sigma}}{d_{\tau(\sigma)}} \begin{cases}A_{i j}^{\sigma} & \text { if } 1 \leqslant i, j \leqslant d_{\sigma} \\ 0 & \text { otherwise. }\end{cases}
$$

Given the fact that $\mathrm{S}_{\Gamma}$ is a complete isometry, $\mathcal{L}^{1}\left(\Sigma_{1}\right)$ contains $\mathcal{L}^{1}(\Gamma)$ 1-uniformly (where $\Gamma \subset \Sigma_{2}$ ). Similarly, using the unboundedness of $\boldsymbol{d}_{\Sigma_{2}}$, we can see that $\mathcal{L}^{1}\left(\Sigma_{2}\right)$ contains $\mathcal{L}^{1}(\Gamma)$ 1-uniformly $\left(\Gamma \subset \Sigma_{1}\right)$. This completes the proof.

Remark 6.2. Given two sets of parameters $\left(\Sigma_{1}, \boldsymbol{d}_{\Sigma_{1}}\right)$ and $\left(\Sigma_{2}, \boldsymbol{d}_{\Sigma_{2}}\right)$, we shall say that $\Sigma_{1} \leqslant \Sigma_{2}$ if there exists an injective mapping $j: \Sigma_{1} \rightarrow \Sigma_{2}$ such that $d_{\sigma} \leqslant d_{j(\sigma)}$ for all $\sigma \in \Sigma_{1}$. Then, by similar arguments to those used in Proposition 6.1, it is easy to see that $B_{\Sigma_{1}}$-convexity is stronger than $B_{\Sigma_{2}}$-convexity whenever $\Sigma_{1} \leqslant \Sigma_{2}$. In particular, if $\Sigma_{0}$ stands for the classical set of parameters, a $B_{\Sigma_{0}}$-convex operator space is automatically $B_{\Sigma}$-convex for any other set of parameters $\Sigma$.

Proposition 6.1 is only a small step in order to see the independence of the notion of $B_{\Sigma}$-convexity with respect to $\Sigma$. However, the general case seems to be more complicated. Now, we give two different conditions which could be useful to decide whether or not the independence with respect to $\Sigma$ holds.

(A) On the notion of $K_{\Sigma^{-}}$-convexity. In order to introduce the notion of $K_{\Sigma^{-}}$ convexity, we need to define the quantized version of the Gauss system. It was already defined by Marcus and Pisier in [8]. More pertinently to the present paper, this system was also treated in the operator space version of Kwapień's Theorem (see [2]). Given a set of parameters $\left(\Sigma, \boldsymbol{d}_{\Sigma}\right)$, we consider a family of independent standard complexvalued Gaussian random variables $\left\{\gamma_{i j}^{\sigma}: \Omega \rightarrow \mathbb{C}: \sigma \in \Sigma, 1 \leqslant i, j \leqslant d_{\sigma}\right\}$ indexed by $\Sigma$ 
and $\boldsymbol{d}_{\Sigma}$. Then, if we construct the random matrices

$$
\gamma^{\sigma}=\frac{1}{\sqrt{d_{\sigma}}}\left(\gamma_{i j}^{\sigma}\right)
$$

we obtain the quantized Gaussian system of parameters $\left(\Sigma, \boldsymbol{d}_{\Sigma}\right)$. On the other hand, given an operator space $E$ and $f \in L_{E}^{2}(\Omega)$, we can consider the Fourier coefficients of $f$ with respect to this system:

$$
\hat{f}(\sigma)=\int_{\Omega} f(\omega) \gamma^{\sigma}(\omega)^{\star} \mathrm{d} \mu(\omega) \in M_{d_{\sigma}} \otimes E .
$$

We shall say that an operator space $E$ is $K_{\Sigma^{-}}$-convex if the Gaussian projection defined as follows

$$
f \in L_{E}^{2}(\Omega) \mapsto \sum_{\sigma \in \Sigma} d_{\sigma} \operatorname{tr}\left(\hat{f}(\sigma) \gamma^{\sigma}\right) \in L_{E}^{2}(\Omega)
$$

is a completely bounded mapping. However, it is obvious that

$$
\sum_{\sigma \in \Sigma} d_{\sigma} \operatorname{tr}\left(\hat{f}(\sigma) \gamma^{\sigma}\right)=\sum_{\sigma \in \Sigma} \sum_{i, j=1}^{d_{\sigma}} \int_{\Omega} f(\omega) \overline{\gamma_{i j}^{\sigma}(\omega)} \mathrm{d} \mu(\omega) \gamma_{i j}^{\sigma},
$$

where $\gamma_{i j}^{\sigma}$ are independent standard complex-valued Gaussian random variables. Hence, it turns out that the notion of $K_{\Sigma^{-}}$-convexity does not depend on the set of parameters $\Sigma$. Moreover, if $\Sigma_{0}$ stands for the classical set of parameters, then any operator space $E$ satisfies

$$
\begin{aligned}
E K_{\Sigma^{-} \text {-convex }} & \Longleftrightarrow E K_{\Sigma_{0}} \text {-convex } \\
& \Longleftrightarrow S^{2}(E) K \text {-convex as a Banach space } \\
& \Longleftrightarrow S^{2}(E) B \text {-convex as a Banach space } \\
& \Longleftrightarrow E B_{\Sigma_{0}} \text {-convex. }
\end{aligned}
$$

Therefore, it follows that the notion of $B_{\Sigma}$-convexity does not depend on the set of

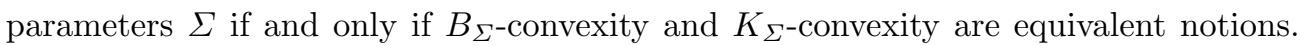
In particular, it provides a possible approach to check this independence. That is, the problem is to generalize to the operator space setting Pisier's Theorem which shows that $K$-convex and $B$-convex Banach spaces are the same (see [12] for more details on this topic).

(B) On how $\boldsymbol{S}_{n}^{\mathbf{1}}$ embeds in $\boldsymbol{S}^{\mathbf{2}}\left(\boldsymbol{l}^{\mathbf{1}}\right)$. Let us suppose that we are given a subspace $F_{n}$ of $S^{2}\left(l_{n^{2}}^{1}\right)$ and a linear isomorphism $\Phi_{n}: S_{n}^{1} \rightarrow F_{n}$ for each $n \geqslant 1$. Let us denote by $F_{n}^{\min }$ the subspace $F_{n}$ equipped with the operator space structure inherited as a subspace of $S^{2}\left(\min l_{n^{2}}^{1}\right)$. Then, if we write $\Psi_{n}: F_{n}^{\min } \rightarrow S_{n}^{1}$ for the inverse of $\Phi_{n}$ with the modified operator space structure on $F_{n}$, we claim that the condition

$$
\left\|\Phi_{n}\right\|_{\mathrm{cb}}\left\|\Psi_{n}\right\| \leqslant k \text { for all } n \geqslant 1 \text { and some constant } k>1
$$


implies the $\Sigma$-independence of $B_{\Sigma}$-convexity. Namely, let $E$ be an operator space which contains $l_{n}^{1}$ uniformly in the sense of Definition 2.5. That is, for all $n \geqslant 1$ there exists a subspace $K_{n}$ of $S^{2}(E)$ and a linear isomorphism $\Lambda_{n}: l_{n}^{1} \rightarrow K_{n}$ such that $\left\|\Lambda_{n}\right\|_{\mathrm{cb}}\left\|\Lambda_{n}^{-1}\right\| \leqslant \lambda$. Now, if we consider the linear isomorphism

$$
\mathrm{T}_{n}=\left(I_{S^{2}} \otimes \Lambda_{n^{2}}\right) \circ \Phi_{n}: S_{n}^{1} \rightarrow H_{n} \subset S^{2}(E),
$$

the inverse operator factors as follows:

$$
H_{n} \rightarrow F_{n}^{\min } \rightarrow S_{n}^{1}
$$

via decomposition $\mathrm{T}_{n}^{-1}=\Psi_{n} \circ\left(I_{S^{2}} \otimes \Lambda_{n^{2}}^{-1}\right)$. In summary, using the well-known properties of the minimal operator space structure, the following estimate follows from condition (6.1):

$$
\left\|\mathrm{T}_{n}\right\|_{\mathrm{cb}}\left\|\mathrm{T}_{n}^{-1}\right\| \leqslant\left\|\Lambda_{n^{2}}\right\|_{\mathrm{cb}}\left\|\Phi_{n}\right\|_{\mathrm{cb}}\left\|\Psi_{n}\right\|\left\|\Lambda_{n^{2}}^{-1}\right\| \leqslant k \lambda .
$$

This gives that $E$ contains $\mathcal{L}^{1}(\Gamma)$ uniformly for any set of parameters $\left(\Sigma, \boldsymbol{d}_{\Sigma}\right)$. There-

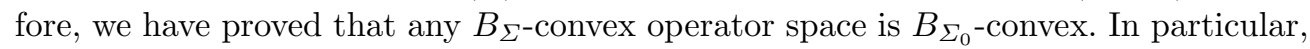

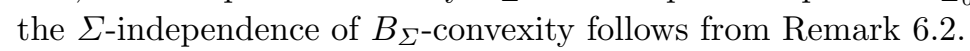

Remark 6.3. As in the Banach space case, the given definition of $K_{\Sigma}$-convexity should not change if we consider the Gaussian projection on $L_{E}^{p}(\Omega)$ instead of $L_{E}^{2}(\Omega)$ for any $1<p<\infty$. Fortunately, this is the case. However, this time the proof cannot be supported by the Khintchine-Kahane inequalities as was explained in Remark 2.3. Nevertheless, the argument is simple. Namely, if we refer to this a priori new notion as $K_{\Sigma}^{p}$-convexity, then an operator space $E$ is $K_{\Sigma^{2}}^{p}$-convex if and only if it is $K_{\Sigma_{0}}^{p}$-convex. But this last condition means that $S^{p}(E)$ is $K$-convex as a Banach space. Now, since $K$-convex and $B$-convex Banach spaces are the same, we conclude that $E$ is $K_{\Sigma}^{p}$-convex if and only if $S^{p}(E)$ is $B$-convex as a Banach space. But, by Corollary 3.4, we know that this is equivalent to saying that $E$ is $B_{\Sigma_{0}}$-convex. Finally, since $K_{\Sigma^{-} \text {-convexity is }}$ equivalent to $B_{\Sigma_{0}}$-convexity, we are done.

Remark 6.4. Condition (6.1) holds and so Pisier's Theorem on the equivalence between $B$-convex and $K$-convex spaces remains valid in the non-commutative setting. The proof of this fact, which will appear as part of a joint work with Junge [6], became clear after this paper was submitted for publication.

Acknowledgements. The author thanks Marius Junge, Magdalena Musat and Gilles Pisier for many illuminating conversations and some suggestions concerning the content of this paper. Research supported in part by the European Commission via the IHP Network 'Harp' and by MCYT Spain via the Project BFM 2001/0189.

\section{References}

1. A. BECK, A convexity condition in Banach spaces and the strong law of large numbers, Proc. Am. Math. Soc. 13 (1962), 329-334.

2. J. García-Cuerva And J. PARCET, Quantized orthonormal systems: a non-commutative Kwapień theorem, Studia Math. 155 (2003), 273-294. 
3. J. García-Cuerva AND J. PARCET, Vector-valued Hausdorff-Young inequality on compact groups, Proc. Lond. Math. Soc., in press.

4. J. García-Cuerva, J. M. Marco and J. Parcet, Sharp Fourier type and cotype with respect to compact semisimple Lie groups, Trans. Am. Math. Soc. 355 (2003), 3591-3609.

5. D. P. GIEsY, On a convexity condition in normed linear spaces, Trans. Am. Math. Soc. 125 (1966), 114-146.

6. M. Junge And J. PARCET, The norm of sums of independent non-commutative random variables in $L_{p}\left(\ell_{1}\right)$, in preparation.

7. S. KWAPIEN, Isomorphic characterizations of inner product spaces by orthogonal series with vector valued coefficients, Studia Math. 44 (1972), 583-595.

8. M. B. MARCUS AND G. PISIER, Random fourier series with applications to harmonic analysis, Annals of Mathematics Studies, vol. 101 (Princeton University Press, 1981).

9. B. MAUREy AND G. Pisier, Séries de variables aléatoires vectorielles indépendantes et propriétés géométriques des espaces de Banach, Studia Math. 58 (1976), 45-90.

10. A. Pietsch And J. Wenzel, Orthonormal systems and Banach space geometry, Encyclopedia of Mathematics and its Applications, vol. 70 (Cambridge University Press, 1998).

11. G. PISIER, Sur les espaces qui ne contiennent pas de $l_{1}^{n}$ uniformément, Séminaire MaureySchwartz 1973/74, Exposé 7 (École Polytechnique, Paris, 1975).

12. G. PISIER, Holomorphic semi-groups and the geometry of Banach spaces, Ann. Math. 115 (1982), 375-392.

13. G. PISIER, The operator Hilbert space $\mathrm{OH}$, complex interpolation and tensor norms, Mem. Am. Math. Soc. 122 (1996), 1-103.

14. G. PISIER, Non-commutative vector valued $L_{p}$-spaces and completely $p$-summing maps, Astérisque (Soc. Math. France) 247 (1998), 1-111. 